\title{
Scaling in Dynamic Susceptibility of Herbertsmithite and Heavy-Fermion Metals
}

\author{
V. R. Shaginyan ${ }^{*},{ }^{1,2}$ A. Z. Msezane,${ }^{2}$ K. G. Popov, ${ }^{3}$ and V. A. Khodel ${ }^{4,5}$ \\ ${ }^{1}$ Petersburg Nuclear Physics Institute, Gatchina, 188300, Russia \\ ${ }^{2}$ Clark Atlanta University, Atlanta, GA 30314, USA \\ ${ }^{3}$ Komi Science Center, Ural Division, RAS, Syktyvkar, 167982, Russia \\ ${ }^{4}$ Russian Research Center Kurchatov Institute, Moscow, 123182, Russia \\ ${ }^{5}$ McDonnell Center for the Space Sciences $\&$ Department of Physics, \\ Washington University, St. Louis, MO 63130, USA
}

\begin{abstract}
We present a theory of the dynamic magnetic susceptibility of quantum spin liquid. The obtained results are in good agreement with experimental facts collected on herbertsmithite $\mathrm{ZnCu}_{3}(\mathrm{OH})_{6} \mathrm{Cl}_{2}$ and on heavy-fermion metals, and allow us to predict a new scaling in magnetic fields in the dynamic susceptibility. Under the application of strong magnetic fields quantum spin liquid becomes completely polarized. We show that this polarization can be viewed as a manifestation of gapped excitations when investigating the spin-lattice relaxation rate.
\end{abstract}

PACS numbers: 75.40.Gb, 64.70.Tg, 76.60.Es, 71.10.Hf

Key Words: Quantum phase transitions; Strongly correlated spin liquid; Heavy fermions; Dynamic magnetic susceptibility; Spin-lattice relaxation rate

\section{INTRODUCTION}

Landau Fermi liquid (LFL) theory is highly successful in the condensed matter physics. The key point of this theory is the existence of fermionic quasiparticles defining the thermodynamic, relaxation and dynamic properties of the material. However, strongly correlated Fermi systems encompassing a variety of systems that display behavior not easily understood within the Fermi liquid theory and called non-Fermi liquid (NFL) behavior. A paradigmatic example of the NFL behavior is represented by heavy-fermion (HF) metals, where a quantum phase transition (QPT) induces a transition between LFL and NFL [1, 2]. QPT can be tuned by different parameters, such as the chemical composition, the pressure, and the magnetic field. Magnetic materials, in particular copper oxides and organic insulators, are interesting subjects of study due to a quantum spin liquid (QSL) that can emerge when they approach QPT and are cooled to low temperature $T$. Exotic QSL is formed with such hypothetic particles as fermionic spinons carrying spin $1 / 2$ and no charge. A search for the materials is a challenge for condensed matter physics [3]. The experimental studies of herbertsmithite $\mathrm{ZnCu}_{3}(\mathrm{OH})_{6} \mathrm{Cl}_{2}$ and the organic insulator $\mathrm{EtMe}_{3} \mathrm{Sb}\left[\mathrm{Pd}(\mathrm{dmit})_{2}\right]_{2}$ have discovered gapless excitations, analogous to excitations near the Fermi surface in HF metals, indicating that $\mathrm{ZnCu}_{3}(\mathrm{OH})_{6} \mathrm{Cl}_{2}$ and $\mathrm{EtMe}_{3} \mathrm{Sb}\left[\mathrm{Pd}(\mathrm{dmit})_{2}\right]_{2}$ are the promising systems to investigate their QPTs and QSLs [4 14]. The observed behavior of the thermodynamic properties of $\mathrm{ZnCu}_{3}(\mathrm{OH})_{6} \mathrm{Cl}_{2}$ strongly resembles that in $\mathrm{HF}$ metals since a simple kagome lattice being strongly frustrated has a dispersionless topologically protected branch of the

\footnotetext{
*E-mail addresses: vrshag@thd.pnpi.spb.ru
}

spectrum with zero excitation energy [14 17]. This indicates that QSL formed by the ideal kagome lattice is located on the ordered side of the fermion condensation quantum phase transition (FCQPT) that is characterized by the presence of the spectrum with zero excitation energy [2]. This observation allows us to establish a close connection between QSL and HF metals whose HF systems are located near FCQPT and, therefore, exhibiting an universal scaling behavior [2, 14, 17]. As we are dealing with the real $3 \mathrm{D}$ compound $\mathrm{ZnCu}_{3}(\mathrm{OH})_{6} \mathrm{Cl}_{2}$ rather than with the ideal 2D kagome lattice, we have to bear in mind that the magnetic interactions and the presence of layers of nonmagnetic $\mathrm{Zn}^{2+}$ ions separating magnetic kagome planes in the substance can shift the QSL from the initial point, positioning it in front of or behind FCQPT. Therefore, the actual location has to be established by analyzing the experimental data. As a result, the location coincides with that of HF metals, and turns out to be at FCQPT [14, 17], as it is shown in Fig. 1. Thus, FCQPT can be considered as QPT of $\mathrm{ZnCu}_{3}(\mathrm{OH})_{6} \mathrm{Cl}_{2}$ QSL and both herbertsmithite and HF metals can be treated in the same framework, so that QSL is composed of fermions and these with zero charge and spin $\sigma= \pm 1 / 2$ occupy the corresponding Fermi sphere with the Fermi momentum $p_{F}[2,14$, 17]. The ground state energy $E(n)$ is given by the Landau functional depending on the quasiparticle distribution function $n_{\sigma}(\mathbf{p})$, where $\mathbf{p}$ is the momentum. In spite of numerous experimental facts collected in measurements of inelastic neutron scattering spectrum and spin-lattice relaxation rates on herbertsmithite, a theoretical understanding of how the dynamical spin susceptibility of QSL behaves on approaching QPT and how it is affected by external parameters, such as the magnetic field, is still missing.

In this letter we employ the Landau transport equation to construct the dynamical spin susceptibility. We 
elucidate how the calculated susceptibility is affected by magnetic field and describe experimental facts collected on herbertsmithite and heavy-fermion metals. The obtained results are in good agreement with the facts and allow us to predict a new scaling emerging under the application of magnetic field in the dynamic susceptibility. Taking into account that QSL becomes completely polarized in strong magnetic fields, we show that this polarization can be seen as the presence of gapped excitations when investigating the spin-lattice relaxation rate.

\section{DYNAMIC SPIN SUSCEPTIBILITY OF QUANTUM SPIN LIQUID AND HEAVY-FERMION METALS}

To construct the dynamic spin susceptibility $\chi(\mathbf{q}, \omega, T)=\chi^{\prime}(\mathbf{q}, \omega, T)+i \chi^{\prime \prime}(\mathbf{q}, \omega, T)$ as a function of momentum $q$, frequency $\omega$ and and temperature $T$, we use the model of homogeneous HF liquid located near FCQPT [2]. To deal with the dynamic properties of Fermi systems, one can use the transport equation describing a slowly varying disturbance $\delta n_{\sigma}(\mathbf{q}, \omega)$ of the quasiparticle distribution function $n_{0}(\mathbf{p})$, and $n=\delta n+n_{0}$. We consider the case when the disturbance is induced by the application of external magnetic field $B=B_{0}+\lambda B_{1}(\mathbf{q}, \omega)$ with $B_{0}$ being a static field and $\lambda B_{1}$ a $\omega$-dependent field with $\lambda \rightarrow 0$. As long as the transferred energy $\omega<q p_{F} / M^{*}<<\mu$, where $M^{*}$ is the effective mass and $\mu$ is the chemical potential, the quasiparticle distribution function $n(\mathbf{q}, \omega)$ satisfies the transport equation 18]

$$
\begin{aligned}
& \left(\mathbf{q} \mathbf{v}_{\mathbf{p}}-\omega\right) \delta n_{\sigma}-\mathbf{q v}_{\mathbf{p}} \frac{\partial n_{0}}{\partial \varepsilon_{p}} \sum_{\sigma_{1} \mathbf{p}_{1}} f_{\sigma, \sigma_{1}}\left(\mathbf{p p}_{1}\right) \delta n_{\sigma_{1}}\left(\mathbf{p}_{1}\right) \\
= & \mathbf{q v}_{\mathbf{p}} \frac{\partial n_{0}}{\partial \varepsilon_{p}} \sigma \mu_{B}\left(B_{0}+\lambda B_{1}\right) .
\end{aligned}
$$

Here $\mu_{B}$ is the Bohr magneton and $\varepsilon_{p}$ is the singleparticle spectrum. We assume that $B_{0}$ is finite but not so strong to lead to the full polarization of the corresponding quasiparticle band. In the field $B_{0}$, the two Fermi surfaces are displaced by opposite amounts, $\pm B_{0} \mu_{B}$, and the magnetization $\mathcal{M}=\mu_{B}\left(\delta n_{+}-\delta n_{-}\right)$, where the two spin orientations with respect to the magnetic field are denoted by \pm , and $\delta n_{ \pm}=\sum_{p} \delta n_{ \pm}(\mathbf{p})$. The spin susceptibility $\chi$ is given by $\chi=\partial \mathcal{M} / \partial B_{\left.\right|_{B=B_{0}}}$. In fact, the transport equation (11) is reduced to two equations which can be solved for each direction \pm and allows one to calculate $\delta n_{ \pm}$and the magnetization. The response to the application of $\lambda B_{1}(\mathbf{q}, \omega)$ can be found by expanding the solution of Eq. (11) in a power series with respect to $M^{*} \omega / q p_{F}$. As a result, we obtain the imaginary part of the spin susceptibility

$$
\chi^{\prime \prime}(\mathbf{q}, \omega)=\mu_{B}^{2} \frac{\omega\left(M^{*}\right)^{2}}{2 \pi q} \frac{1}{\left(1+F_{0}^{a}\right)^{2}},
$$

where $F_{0}^{a}$ is the dimensionless spin antisymmetric quasiparticle interaction [18]. The interaction $F_{0}^{a}$ is found to saturate at $F_{0}^{a} \simeq-0.8[19,20]$ so that $\left(1+F_{0}^{a}\right)$ is positive. It is seen from Eq. (2) that the second term is an odd function of $\omega$. Therefore, it does not contribute to the real part $\chi^{\prime}$ and forms the imaginary part $\chi^{\prime \prime}$. Taking into account that at relatively high frequencies $\omega \geq q p_{F} / M^{*} \ll \mu$ in the hydrodynamic approximation $\chi^{\prime} \propto 1 / \omega^{2}$ [21], we conclude that the equation

$$
\chi(\mathbf{q}, \omega)=\frac{\mu_{B}^{2}}{\pi^{2}\left(1+F_{0}^{a}\right)} \frac{M^{*} p_{F}}{1+i \pi \frac{M^{*} \omega}{q p_{F}\left(1+F_{0}^{a}\right)}},
$$

produces the simple approximation for the susceptibility $\chi$ and satisfies the Kramers-Kronig relation connecting the real and imaginary parts of $\chi$.

To understand how can $\chi^{\prime \prime}$ and $\chi$ given by Eqs. (2) and (3), respectively, depend on temperature $T$ and magnetic field $B$, we recall that near FCQPT point the effective mass $M^{*}$ depends on $T$ and $B$, and is given by the Landau equation (LE) 2, 22]. The interaction function $F$ of $\mathrm{LE}$ is completely defined by the fact that the system has to be at FCQPT. The sole role of $F$ is to bring the system to FCQPT, where the Fermi surface alters its topology so that $M^{*}$ acquires $T$ and $B$ dependencies [2, 23, 24]. At FCQPT, LE can be solved analytically: At $B=0$, the effective mass depends on $T$

$$
M^{*}(T) \simeq a_{T} T^{-2 / 3} .
$$

At finite $T$, the application of magnetic field $B$ drives the system to the LFL region with

$$
M^{*}(B) \simeq a_{B} B^{-2 / 3} .
$$

Here $a_{T}$ and $a_{B}$ are constants. At finite $B$ and $T$ near FCQPT, the solutions of LE can be well approximated by a simple universal interpolating function. The interpolation occurs between the LFL $\left(M^{*}(T) \propto\right.$ const $)$ and NFL $\left(M^{*}(T) \propto T^{-2 / 3}\right)$ regions. It is convenient to introduce the normalized effective mass $M_{N}^{*}$ and the normalized temperature $T_{N}$ dividing the effective mass $M^{*}$ by its maximal values, $M_{\max }^{*}$, and temperature $T$ by $T_{\max }$ at which the maximum occurs. The normalized effective mass $M_{N}^{*}=M^{*} / M_{\max }^{*}$ as a function of the normalized temperature $y=T_{N}=T / T_{\max }$ is given by the interpolating function [2]

$$
M_{N}^{*}(y) \approx c_{0} \frac{1+c_{1} y^{2}}{1+c_{2} y^{8 / 3}} .
$$

Here $c_{0}=\left(1+c_{2}\right) /\left(1+c_{1}\right), c_{1}$ and $c_{2}$ are fitting parameters, making $M_{N}^{*}(y=1)=1$. Magnetic field $B$ enters LE only in the combination $\mu_{B} B / k_{B} T$, making $k_{B} T_{\max } \simeq \mu_{B} B$ where $k_{B}$ is the Boltzmann constant 2, 23]. Thus, in the presence of magnetic fields the variable $y$ becomes $y=T / T_{\max } \simeq k_{B} T / \mu_{B} B$. Since the variables $T$ and $B$ enter symmetrically Eq. ([6) is valid for 


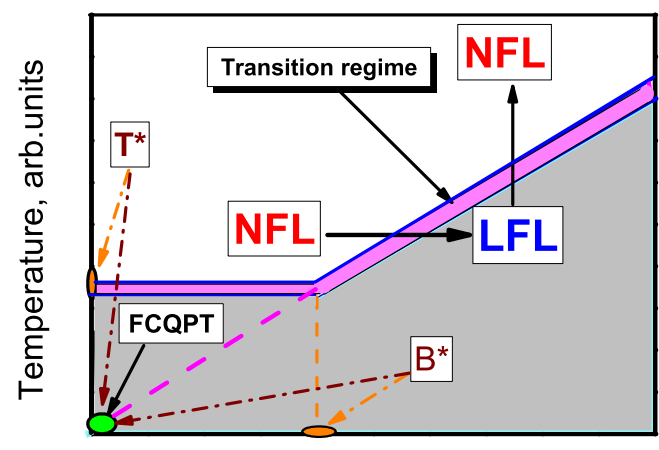

Control parameter, magnetic field B

FIG. 1: (color online). $T-B$ phase diagram of QSL and HF liquid. The vertical and horizontal arrows, crossing the transition region depicted by the thick lines, show LFL-NFL and NFL-LFL transitions at fixed $B$ and $T$, respectively. At temperatures $T<T^{*}$ and magnetic field $B<B^{*}$ shown by the dash-dot arrows the effective mass $M^{*} \simeq$ const and the system in the LFL region. The dash line continuing the thick line represents the transition region provided the system were located at FCQPT shown by the arrow.

$y=\mu_{B} B / k_{B} T$. Now we construct the schematic $T-B$ phase diagram of QSL and HF liquid reported in Fig. 1. At $T=0$ and $B=0$ the system can exactly be located at the FCQPT point without tuning with both $T^{*}$ and $B^{*}$ are zero. It can also be shifted from the FCQPT point by doping, pressure etc. In that case $T^{*}$ and $B^{*}$ become finite so that at $T<T^{*}$ and $B<B^{*}$ the effective mass $M^{*} \simeq$ const. As seen from Fig. 1, at $T \simeq T^{*}$ and $B \simeq B^{*}$ the transition region exhibits a kink, since $M^{*}$ is no longer constant at rising $B$ and $T$. Magnetic field $B$ and temperature $T$ play the role of the control parameters, driving it from the NFL to LFL regions as shown by the vertical and horizontal arrows. At fixed $B$ and increasing $T$ the system transits along the vertical arrow from the LFL region to NFL one crossing the transition region. On the contrary, at fixed $T$ increasing $B$ drives the system along the horizontal arrow from the NFL region to LFL one.

\section{SCALING BEHAVIOR OF THE DYNAMIC SUSCEPTIBILITY}

To elucidate a scaling behavior of $\chi$, we employ Eq. (4) to describe the temperature dependence of $\chi$. It follows from Eqs. (3) and (4) that

$$
T^{2 / 3} \chi(T, \omega) \simeq \frac{a_{1}}{1+i a_{2} E} .
$$

Here $a_{1}$ and $a_{2}$ are constants absorbing irrelevant values and $E=\omega /\left(k_{B} T\right)^{2 / 3}$. As a result, the imaginary part
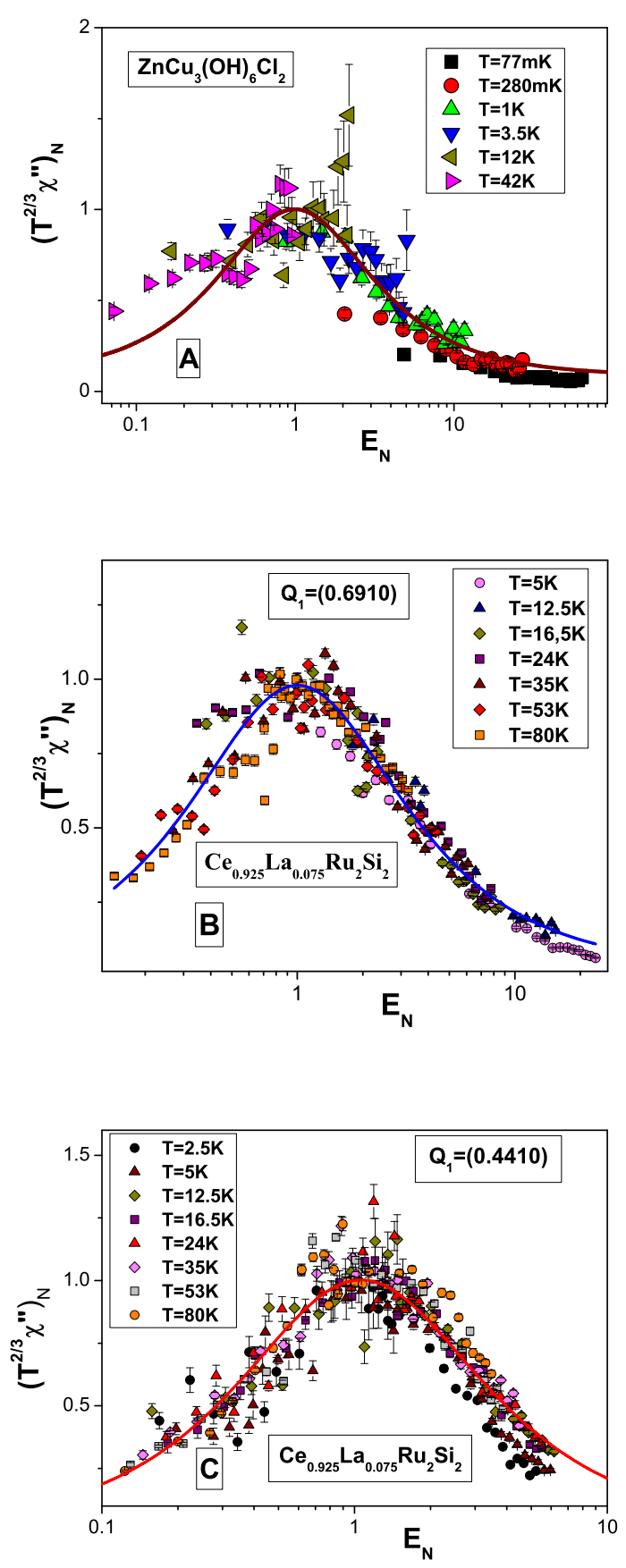

FIG. 2: (color online). The function $\left(T^{2 / 3} \chi^{\prime \prime}\right)_{N}$ plotted against the unitless ratio $E_{N}=\omega /\left(\left(k_{B} T\right)^{2 / 3} E_{\max }\right)$. The data extracted from measurements on $\mathrm{ZnCu}_{3}(\mathrm{OH})_{6} \mathrm{Cl}_{2}$ obtained for $0.077<T<42 \mathrm{~K}$ [7], Panel A, and on the HF metal $\mathrm{Ce}_{0.925} \mathrm{La}_{0.075} \mathrm{Ru}_{2} \mathrm{Si}_{2}$ obtained for $2.5<T<80 \mathrm{~K}$ at $Q_{1}[25]$, Panel B and C, collapse onto a single curve. The solid curves are fits with the function given by Eq. (9). 
$\chi^{\prime \prime}(T, \omega)$ satisfies the equation

$$
T^{2 / 3} \chi^{\prime \prime}(T, \omega) \simeq \frac{a_{3} E}{1+a_{4} E^{2}},
$$

where $a_{3}$ and $a_{4}$ are constants. It is seen from Eq. (8) that $T^{2 / 3} \chi^{\prime \prime}(T, \omega)$ has a maximum $\left(T^{2 / 3} \chi^{\prime \prime}(T, \omega)\right)_{\max }$ at some $E_{\max }$ and depends on the only variable $E$. Equation (8) confirms the scaling behavior of $\chi^{\prime \prime} T^{0.66}$ experimentally established in Ref. [7]. As it was done for the effective mass when constructing (6), we introduce the dimensionless function $\left(T^{2 / 3} \chi^{\prime \prime}\right)_{N}=T^{2 / 3} \chi^{\prime \prime} /\left(T^{2 / 3} \chi^{\prime \prime}\right)_{\max }$ and the dimensionless variable $E_{N}=E / E_{\max }$, and Eq. (8) is modified to read

$$
\left(T^{2 / 3} \chi^{\prime \prime}\right)_{N} \simeq \frac{b_{1} E_{N}}{1+b_{2} E_{N}^{2}},
$$

with $b_{1}$ and $b_{2}$ are fitting parameters which are to adjust the function on the right-hand side of Eq. (9) to reach its maximum value 1 at $E_{n}=1$. We predict that if measurements of $\chi^{\prime \prime}$ are taken at fixed $\mathrm{T}$ as a function of $B$, then taking into account Eq. (5), we again obtain that the function $B^{2 / 3} \chi^{\prime \prime}(E)$ exhibits the scaling behavior with $E=\omega /\left(\mu_{B} B^{2 / 3}\right)$. If the system is placed at FCQPT, the scaling described above is valid down to lowest temperatures. When the system is shifted from FCQPT then $T^{*}$ and $B^{*}$ are finite and the scaling is violated in the LFL region and recovered in the NFL one at $T>T^{*}$ or $B>B^{*}$ as seen from Fig. 1,

In Fig. 2 consistent with Eq. (9), the scaling of the normalized dynamic susceptibility $\left(T^{2 / 3} \chi^{\prime \prime}\right)_{N}$ extracted from the inelastic neutron scattering spectrum of both herbertsmithite 7], Panel A, and $\mathrm{Ce}_{0.925} \mathrm{La}_{0.075} \mathrm{Ru}_{2} \mathrm{Si}_{2}$, Panel B and C, 25] is displayed. The scaled data collapse fairly well onto a single curve over almost three decades of $E_{N}$. It is seen that our calculations shown by the solid curves are overall in good agreement with the experimental facts. We note that, as seen from Fig. 2 Panel $\mathrm{C}$, the data taken at $2.5 \mathrm{~K}$ are at variance with the scaling behavior shown by the solid curve. We suggest that $\mathrm{Ce}_{0.925} \mathrm{La}_{0.075} \mathrm{Ru}_{2} \mathrm{Si}_{2}$ is slightly shifted from FCQPT as shown in Fig. 1 and at sufficiently low temperatures $T<T^{*}$ the scaling is violated [25, 26], while $\mathrm{ZnCu}_{3}(\mathrm{OH})_{6} \mathrm{Cl}_{2}$ is near that point. Some remarks on a role of both the disorder and the anisotropy are in order. The anisotropy is supposed to be related to the Dzyaloshinskii-Moriya interaction, exchange anisotropy, or out-of-plane impurities. Measurements of the susceptibility on the single crystal of herbertsmithite have shown that it closely follows that measured on a powder sample [8]. At low temperatures $T \lesssim 70 \mathrm{~K}$, the single-crystal data do not show magnetic anisotropy [8]. These confirm that the stoichiometry, disorder and anisotropy do not contribute significantly to the results at relatively low temperatures. Moreover, the scaling behavior of the thermodynamic functions of herbertsmithite is the intrinsic feature and has nothing to do with the impurities
17]. These observations are in agreement with a general consideration of scaling behavior of HF metals [2].

\section{SPIN-LATTICE RELAXATION RATE OF QUANTUM SPIN LIQUID}

Consider the effect of $B$ on the spin-lattice relaxation rate $1 / T_{1} T$ determined by $\chi^{\prime \prime}$ given by Eq. (2)

$$
\frac{1}{T_{1} T}=\left.\frac{3}{4 \mu_{B}^{2}} \sum_{\mathbf{q}} A_{\mathbf{q}} A_{-\mathbf{q}} \frac{\chi^{\prime \prime}(\mathbf{q}, \omega)}{\omega}\right|_{\omega \rightarrow 0} \propto\left(M^{*}\right)^{2},
$$

where $A_{\mathbf{q}}$ is the hyperfine coupling constant of the muon (or nuclei) with the spin excitations at wave vector $\mathbf{q}$ 2, 27, 28]. Figure 3 and the inset display the normalized $\left(1 / T_{1} T\right)_{N}$ and the normalized longitudinal magnetoresistance $\rho_{N}$ at fixed temperature versus the normalized magnetic field $B_{N}$. It is seen from Fig. 3 that the magnetic field progressively reduces $1 / T_{1} T$ and the longitudinal magnetoresistance (LMR), and these as a function of $B$ possess an inflection point at $B=B_{\text {inf }}$ shown by the arrow. The normalized LMR obeys the equation [2]

$$
\rho_{N}\left(B_{N}\right)=\frac{\rho\left(B_{N}\right)-\rho_{0}}{\rho_{\text {inf }}}=\left(\frac{1}{T_{1} T}\right)_{N}=\left(M_{N}^{*}\right)^{2},
$$

where $\rho_{0}$ is the residual resistance, $\rho_{\text {inf }}$ is LMR taken at the inflection point, $\rho$ is LMR, and $B_{N}=B / B_{\text {inf }}$. We normalize $\left(1 / T_{1} T\right)$ and LMR by their values at the inflection point, and the magnetic field is normalized by $B_{\text {inf }}$. In accordance with the phase diagram 1 at $B>B_{\text {inf }}$, as seen from Fig. 3. QSL enters the LFL region with $B$-dependence of the effective mass defined by Eq. (5). It follows from Eqs. (10) and (11) that $\left(1 / T_{1} T\right)_{N}=\rho_{N}=\left(M_{N}^{*}\right)^{2}$ where $\left(M_{N}^{*}\right)^{2}$ is defined by Eq. (6) which shows that different strongly correlated Fermi systems are to exhibit the same scaling of $\left(M_{N}^{*}\right)^{2}$. It is seen from Fig. 3 and from the inset, that $\mathrm{YbCu}_{5-\mathrm{x}} \mathrm{Au}_{\mathrm{x}}$, herbertsmithite $\mathrm{ZnCu}_{3}(\mathrm{OH})_{6} \mathrm{Cl}_{2}$ and $\mathrm{YbRh}_{2} \mathrm{Si}_{2}$ demonstrate the similar behavior of $\left(M_{N}^{*}\right)^{2}$ resulting in the scaling of LMR and $1 / T_{1} T$. Thus, Eqs. (2), (10) and (11) determine the close relationship existing between the quite different dynamic properties and different strongly correlated Fermi systems such as QSL and HF metals, revealing their scaling behavior at FCQPT.

We note that one may be confused when applying Eq. (10) to describe $\left(1 / T_{1} T\right)$ in strong magnetic fields. In that case both QSL and HF metals become fully polarized due to Zeeman splitting [14, 27, 28, 32]. As a result, one subband becomes empty, while the energy $\varepsilon_{F}$ of spinons at the Fermi surface of the other subband lies below the chemical potential $\mu$ formed by the magnetic field $B_{0}$. It follows from Eq. (11) that $\chi^{\prime \prime}=0$ and Eq. (10) is not valid. The difference $\delta=\mu-\varepsilon_{F}$ can be viewed as a gap that makes $1 / T_{1} T \propto \exp -\left(\delta / k_{B} T\right)$. At temperatures $k_{B} T \sim \delta$, the subbands are populated by 


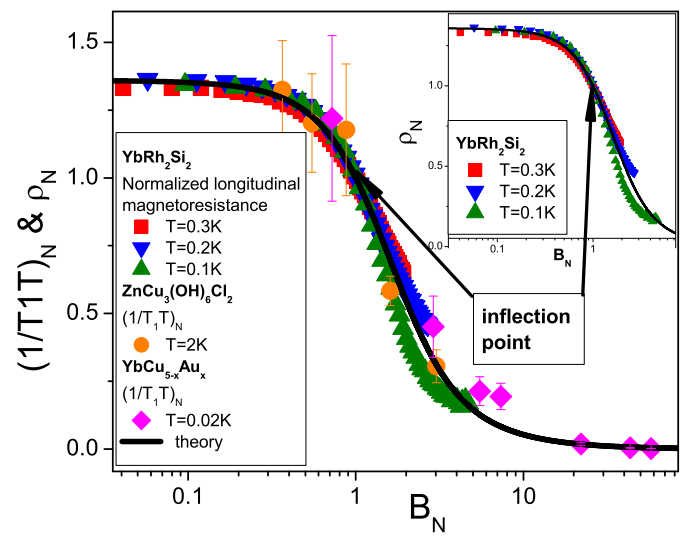

FIG. 3: (color online). Magnetic field dependence of normalized (see text for details) muon spin-lattice relaxation rate $\left(1 / T_{1} T\right)_{N}$ extracted from measurements on $\mathrm{YbCu}_{4.4} \mathrm{Au}_{0.6}[29]$ and $\mathrm{ZnCu}_{3}(\mathrm{OH})_{6} \mathrm{Cl}_{2}$ [30] along with the normalized longitudinal magnetoresistance $\rho_{N}$ versus normalized magnetic field $B_{N}$. Our calculations are shown by the solid line. The arrows indicate the inflection points. Inset: $\rho_{N}$ versus $B_{N}, \rho_{N}$ is extracted from measurements on $\mathrm{YbRh}_{2} \mathrm{Si}_{2}$ at different temperatures 31] listed in the legend. The solid curve represents our calculations.

spinons and the validity of Eq. (10) is restored. Thus, $\delta$ can be interpreted as the presence of gapped excitations. On the other hand, if there were the gapped excitations, then the heat capacity demonstrates the exponential decay rather than a linear $T$-dependence at low temperatures. Analysis based on experimental data shows the presence of linear $T$-dependence even under the application of high magnetic fields [14], while recent measurements on $\mathrm{ZnCu}_{3}(\mathrm{OH})_{6} \mathrm{Cl}_{2}$ of $1 / T_{1} T$ suggest the gapped excitations [33]. To clarify whether the gapped excitations would occur in $\mathrm{ZnCu}_{3}(\mathrm{OH})_{6} \mathrm{Cl}_{2}$, an accurate experimental measurement in magnetic fields of the low temperature heat capacity is necessary.

\section{CONCLUSIONS}

We have presented a theory of the dynamic magnetic susceptibility of quantum spin liquid, and elucidated how the calculated susceptibility is affected by magnetic field and describe experimental facts collected on herbertsmithite and heavy-fermion metals. The obtained results are in good agreement with experimental facts collected on both herbertsmithite $\mathrm{ZnCu}_{3}(\mathrm{OH})_{6} \mathrm{Cl}_{2}$ and on heavy-fermion metals, and allow us to conclude that the dynamic magnetic susceptibility of herbertsmithite is similar to that of heavy-fermion metals. Thus, herbertsmithite can be viewed as a new type of strongly correlated electrical insulator that possesses properties of heavy-fermion metals with one exception: it resists the flow of electric charge. We have also predicted a new scaling in magnetic fields in the dynamic susceptibility emerging under the application of magnetic field. Taking into account that under the application of strong magnetic fields quantum spin liquid becomes completely polarized, we have shown that this polarization can be viewed as a manifestation of gapped excitations when investigating the spin-lattice relaxation rate.

\section{ACKNOWLEDGEMENTS}

This work was supported by U.S. DOE, Division of Chemical Sciences, Office of Basic Energy Sciences, Office of Energy Research, and AFOSR.

[1] H.v. Löhneysen, A. Rosch, M. Vojta, P. Wölfle, Rev. Mod. Phys. 79 (2007) 1015.

[2] V. R. Shaginyan, M. Ya. Amusia, A. Z. Msezane, K. G. Popov, Phys. Rep. 492 (2010) 31.

[3] L. Balents, Nature 464 (2010) 199.

[4] M. P. Shores, E. A. Nytko, B. M. Bartlett, D. G. Nocera, J. Am. Chem. Soc. 127 (2005) 13462.

[5] J. S. Helton, et al., Phys. Rev. Lett. 98 (2007) 107204.

[6] M. A. deVries, K. V. Kamenev, W. A. Kockelmann, J. Sanchez-Benitez, A. Harrison Phys. Rev. Lett. 100 (2008) 157205.

[7] J. S. Helton, et al., Phys. Rev. Lett. 104 (2010) 147201.

[8] T. H. Han, et al., Phys. Rev. B 83 (2011) 100402(R).

[9] F. Bert, P. Mendels, J. Phys. Soc. Jpn. 79 (2010) 011001.

[10] F. Mila, Phys. Rev. Lett. 81 (1998) 2356.

[11] S. S. Lee, P. A. Lee, Phys. Rev. Lett. 95 (2005) 036403.

[12] M. Yamashita, et al., Science 328 (2010) 1246.

[13] Y. Ran, M. Hermele, P. A. Lee, X. G. Wen, Phys. Rev. Lett. 98 (2007) 117205.

[14] V. R. Shaginyan, A. Z. Msezane, K. G. Popov, Phys. Rev. B 84 (2011) 060401(R).

[15] D. Green, L. Santos, C. Chamon, Phys. Rev. B 82 (2010) 075104.

[16] T. T. Heikkila, N. B. Kopnin, G. E. Volovik, JETP Lett. 94 (2011) 233.

[17] V. R. Shaginyan, A. Z. Msezane, K. G. Popov, G. S. Japaridze, V. A. Stephanovich, Europhys. Lett. 97 (2012) 56001.

[18] D. Pines, P. Noziéres, Theory of Quantum Liquids, Benjamin, New York, 1966.

[19] M. Pfitzner, P. Wölfle, Phys. Rev. B 33 (1986) 2003.

[20] D. Vollhardt, P. Wölfle, P. W. Anderson, Phys. Rev. B 35 (1987) 6703.

[21] D. Forster, Hydrodynamic Fluctuations, Broken Symmetry, and Correlation Functions, W. A. Benjamin, Inc. 1975.

[22] L. D. Landau, Sov. Phys. JETP 3 (1956) 920.

[23] J. W. Clark, V. A. Khodel, M. V. Zverev, Phys. Rev. B 71 (2005) 012401. 
[24] V. A. Khodel, J. W. Clark, M. V. Zverev, Phys. Rev. B 78 (2008) 075120.

[25] W. Knafo, et al., Phys. Rev. B 70 (2004) 174401.

[26] O. Stockert, F. Steglich, Annu. Rev. Condens. Matter Phys. 2 (2011) 79.

[27] J. Koringa, Physica 16 (1950) 601.

[28] T. Moriya, Spin Fluctuations in Itinerant Electron Magnetism, Springer, Berlin, 1985.
[29] P. Carretta, R. Pasero, M. Giovannini, C. Baines, Phys. Rev. B 79 (2009) 020401(R).

[30] T. Imai, E. A. Nytko, B.M. Bartlett, M. P. Shores, D. G. Nocera, Phys. Rev. Lett. 100 (2008) 077203.

[31] P. Gegenwart, et al., Science 315 (2007) 969.

[32] V. R. Shaginyan, et al., Europhys. Lett. 93 (2011) 17008.

[33] M. Jeong, et al., Phys. Rev. Lett. 107 (2011) 237201. 